\title{
Evaluating through mathematical modelling the power equipment busbars electrodynamic strength under sudden short-circuit conditions
}

\author{
Cornelia A. Bulucea ${ }^{1,}$, Constantin Brindusa ${ }^{2}$, Doru A. Nicola ${ }^{1}$, Nikos E. Mastorakis ${ }^{3}$, Carmen A. Bulucea ${ }^{4}$ and Philippe \\ Dondon $^{5}$ \\ ${ }^{1}$ University of Craiova, Faculty of Electrical Engineering, Craiova 200440, Romania \\ ${ }^{2}$ University "Constantin Brancusi" of Targu-Jiu, Targu-Jiu 210135, Romania \\ ${ }^{3}$ Technical University of Sofia, English Language Faculty of Engineering, Sofia 1000, Bulgaria \\ ${ }^{4}$ University of Medicine and Pharmacy of Craiova, Faculty of Medicine, Craiova 200349, Romania \\ ${ }^{5}$ ENSEIRB MATMECA - Bordeaux INP, Domaine Universitaire 33405 TALENCE, France
}

\begin{abstract}
The electrodynamic strength, as forces acting between the current-carrying electric circuits are exerted as long as the currents exist, and have the tendency of deformation and displacement of the circuits. In short-circuit regimes the strength in electrical equipment becomes severe. For instance, short-circuits highly affect power transformers connected to power transmission lines. The effects are also strong because of mechanical deformations occurring in the power transformer connection part. In line with this idea, in this paper it is made an analytical study upon the a.c. single-phase and a.c. three-phase electric circuits, taking into account the current instantaneous maximum value. The paper also entails numerical simulations of electrodynamic strength in power transformer busbars under short-circuit conditions. MATLAB software, with its specific extensions, enable simulation models to generate the charts of the electrodynamic forces in the power transformer connection bars.
\end{abstract}

\section{Introduction}

Electric equipment, specifically power transformers are constantly subjected to specific electrodynamic strengths. In normal operating regimes of electric equipment, the values of the electrodynamic forces are relatively small. But, in the fault regimes the strength values become important and can produce irreversible mechanical damages of the equipments carrying the fault currents [1-8]. The internal connections based on busbars are those where strong mechanical effects may occur under the action of the electrodynamic forces generated by the fault currents, specifically, short-circuits. An analytical study upon the a.c. single-phase and a.c. threephase electric circuits is presented in this paper. Electrodynamic forces modelling in a.c. circuits emphasizes aspects with direct implication on power equipment. Further in this paper there are presented results of numerical simulation of short-circuit regime in the particular case of power transformer busbars, using MATLAB software and its extensions.

\section{Electrodynamic forces in a.c. equipment}

In the alternating current-carrying circuits, the electrodynamic forces must be calculated taking into account the instantaneous values of the currents. Consequently, the electrodynamic forces will be not constant, having a variation with time depending on the currents frequency. The forces maximum value depends on the regime (steady-state or transient) of the electric currents evolution.

Like reference [1-8], it is taken into consideration the force $\mathrm{F}$ between two straight parallel conductors and it is noted by $f_{12}=F /\left(i_{1} i_{2}\right)$ the specific electrodynamic force (on the current unit). The magnitude of the specific force is constant, given by:

$$
f_{12}=\frac{F}{i_{1} i_{2}}=10^{-7} \frac{2 l}{a} k_{s}\left[N / A^{2}\right]
$$

The calculation will be made for the currents in the steady-state regime and the maximum will be extrapolated in relation with the peak values of the short-circuit currents [3-4]. There will be analyzed the electrodynamic forces in the a.c. single-phase circuit and in the a.c. three-phase circuits (depending on the geometrical setting of the phase conductors).

\subsection{Electrodynamic forces in single-phase circuits}

*orresponding author: abulucea@em.ucv.ro 
Between two straight parallel conductors carrying in opposite senses the alternating current $\boldsymbol{i}$ will be exerted the repulsive electrodynamic forces $\vec{F}$, like in Fig.1.

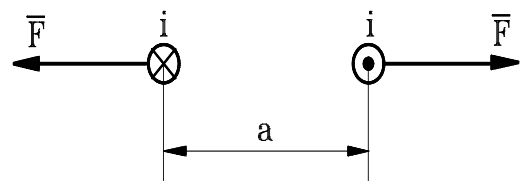

Fig.1. Electrodynamic forces in single-phase circuits

For the alternating currents $i_{1}=i_{2}=i=I_{m} \sin \omega t=\sqrt{2} I \sin \omega t$, the repulsive force between the two parallel conductors takes the form:

$$
F=f_{12} i_{1} i_{2}=f_{I 2}\left(2 I^{2}\right) \sin ^{2} \omega t=\frac{1}{2} f_{12}(1-\cos 2 \omega t)(\sqrt{2} I)^{2}
$$

The expression (2) emphasizes two components of the force $\mathrm{F}$ :

- a constant component, with the magnitude $\frac{1}{2} f_{12}(\sqrt{2} I)^{2}$ and

- a variable component with a cosinusoidal time variation, having the same amplitude as the constant component, but with a frequency of variation that is doubled than the currents frequency. Because $\cos 2 \omega t$ is varying periodically between +1 and -1 , the electrodynamic force $\mathrm{F}$ will have a variation between 0 and $2 \mathrm{~F}_{\mathrm{av}}$, without a sign change. The electrodynamic force is pulsing between 0 and $\mathrm{F}_{\max }$.

The average value of the repulsion electrodynamic force $\mathrm{F}_{\mathrm{av}}$ is given by:

$$
F_{a v}=\frac{1}{2} f_{12}(\sqrt{2} I)^{2}
$$

The maximum value of the electrodynamic force $F_{\max }$ is calculated as follows:

$$
F_{\text {max }}=2 \frac{1}{2} f_{12}(\sqrt{2} I)^{2}=2 F_{a v}
$$

Relation (4) emphasizes that any alternating current with the r.m.s. value I (equal to the direct current I magnitude) is producing a maximum electrodynamic force doubled than the force produced (in the same conditions) by the direct current $\mathrm{I}_{\mathrm{dc}}=\mathrm{I}$.

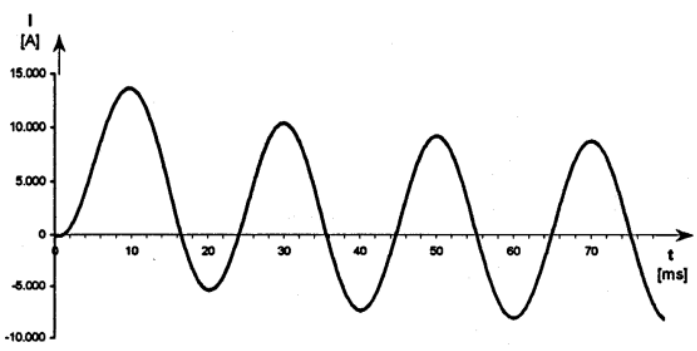

Fig.2. Transient short-circuit current evolution

In the case of the a.c. single-phase circuits submitted at short-circuit conditions, the current $i_{k}$ is modifying with time, becomes asymmetrical [1-8] and the instantaneous maximum value $i_{\text {peak }}$ (the first peak, named also the shock current) is strongly depending on the short-circuit appearance moment and on the circuit parameters, by the ratio $\mathrm{X} / \mathrm{R}[1-8]$.

If it is taken into consideration the value of the peak factor $\chi$, it will be emphasized (see Fig.2) that the asymmetric short-circuit peak current value $i_{\text {peak }}=\sqrt{2} I_{k} \chi$ is greater than the maximum current value in the steady-state short-circuit $\sqrt{2} I_{k}$.

In Fig. 3 there are shown the electrodynamic forces evolutions in the transient short-circuit regimes, for two values of the circuit time constant $\tau$.

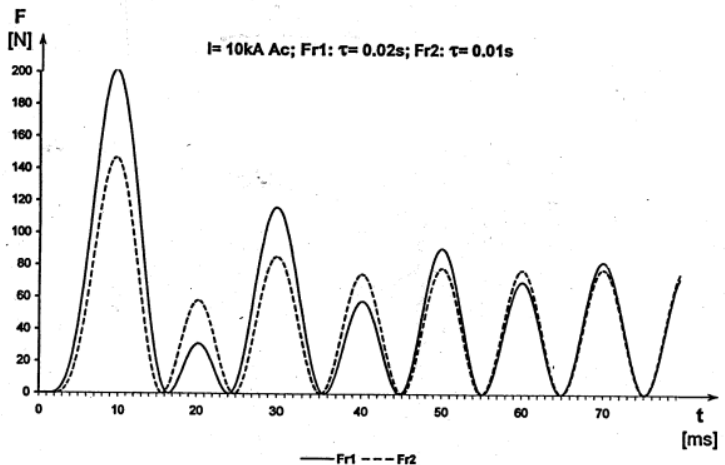

Fig.3. Resultant forces in transient short-circuit regime

When $\chi=1,8$, the short-circuit maximum value of the a.c. electrodynamic force (4) becomes:

$F_{\max (a . c)}=2 \frac{1}{2} f_{12}\left(\sqrt{2} I_{k} 1,8\right)^{2} \approx 6,48\left(f_{12} I_{k}^{2}\right)=6,48 F_{\max (d . c .)}$

\subsection{Electrodynamic forces in three-phase circuits}

These forces are exerting between the phase conductors of the three-phase electric circuits. Particularly, in a shortcircuit regime, the forces can reach important values [1-8].

Apart from the forces in the a.c. single-phase circuits (which always were maintaining the sense), in the threephase circuits a conductor is submitted to a force varying with time, which can shift its sense [1-8], passing from the attraction to the repulsion (and the reverse).

In the paper will be analyzed the influence of the conductors settlemet upon the resultant forces magnitude. There are taken into consideration the currents in a steadystate short-circuit, as the form:

$i_{1}=\sqrt{2} I \sin \omega t ; i_{2}=\sqrt{2} I \sin \left(\omega t-\frac{2 \pi}{3}\right) ; i_{3}=\sqrt{2} I \sin \left(\omega t+\frac{2 \pi}{3}\right)$

\subsubsection{Electrodynamic forces at same plane conductors settlement}

There are considered three-phase conductors, which are straight, parallel and in the same plane, as depicted in Fig.4. For the forces evaluation, conventionally it is established a current sense for the conductor upon is exerted the searched force and there are admitted opposite senses for the other two currents. For instance (Fig.4.a), on 
the first conductor 1 will interact the currents from the conductors 2 and 3 , by the repulsive forces $F_{21}$ and $F_{31}$.

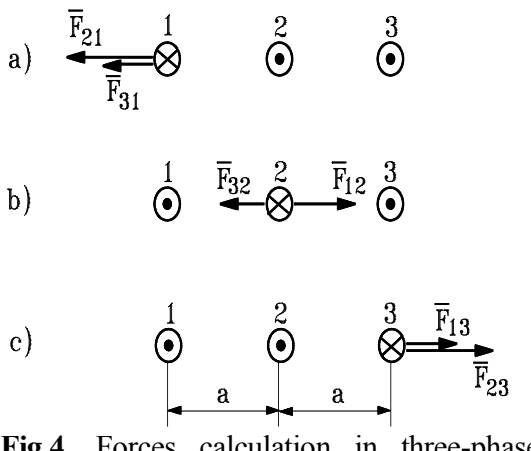

Fig.4. Forces calculation in three-phase circuits with conductors in same plane

The resulting force $\mathrm{F}_{1}$ will be:

$$
F_{1}=F_{21}+F_{31}=f_{21} i_{2} i_{1}+f_{31} i_{3} i_{1}
$$

Depending on the force sign $F_{1}$ it will appear the "conductor 1 repulsion" (if $\mathrm{F}_{1}>0$ ) or the "conductor 1 attraction" (if $\left.\mathrm{F}_{1}<0\right)$.

Based on the specific forces definition relation (1), there are specified that:

$$
f_{21}=f_{12} ; f_{31}=f_{13} ; f_{31}=0,5 f_{21}
$$

Taking into account the relations (8) and the currents expressions from (6), the total force magnitude $F_{1}$ (exerted upon the conductor 1), calculated with (7) will be:

$$
F_{1}=f_{21} i_{1}\left(i_{2}+\frac{1}{2} i_{3}\right)=\frac{\sqrt{3}}{4} f_{12}\left[\sin \left(2 \omega t+\frac{2 \pi}{3}\right)-\frac{\sqrt{3}}{2}\right]\left(2 I^{2}\right)
$$

Thus, in the steady-state regime of the three-phase currents, the force $F_{1}$ has a harmonic time variation, with the doubled currents frequency. Moreover:

- for $\sin \left(2 \omega t+\frac{2 \pi}{3}\right)=+1$ the force $F_{1}$ will have the maximum positive value (it is an "attraction force"), with the magnitude $\mathrm{F}_{\text {latr: }}$ :

$$
F_{\text {latr }}=\frac{\sqrt{3}}{4} f_{12}\left(1-\frac{\sqrt{3}}{2}\right) 2 I^{2}=0,0580 f_{12}\left(2 I^{2}\right)
$$

- for $\sin \left(2 \omega t+\frac{2 \pi}{3}\right)=-1$ the force $\mathrm{F}_{1}$ will have the maximum negative value (it is a "repulsion force"), with the magnitude $\mathrm{F}_{\text {1rep }}$ :

$$
F_{1 \text { rep }}=\frac{\sqrt{3}}{4} f_{12}\left(1+\frac{\sqrt{3}}{2}\right) 2 I^{2}=0,8080 f_{12}\left(2 I^{2}\right)
$$

Similarly, the forces $F_{13}$ and $F_{23}$ which are experienced upon the conductor 3 (see Fig.4.c) have the same magnitude order like those experienced upon the conductor 1 (acting on the same direction with the forces $F_{21}$ and $F_{31}$, but in opposite senses).

The forces $F_{13}$ and $F_{23}$ which are exerted upon the middle conductor 2 (see Fig.4.b) are acting on the same direction, but in opposite senses. Taking as positive the sense of the force $F_{1}$, for the calculation of the force $F_{2}$ it will result:

$$
F_{2}=F_{32}-F_{12}=f_{32} i_{3} i_{2}-f_{12} i_{1} i_{2}
$$

Due to the symmetrical arrangement of the conductors 1 and 3 we have $f_{12}=f_{32}$ and with the currents relations from
(12), the resulting force expression $F_{2}$ can be brought to the form:

$F_{2}=\frac{\sqrt{3}}{2} f_{12}\left[\sin \left(2 \omega t-\frac{\pi}{3}\right)\right]\left(2 I^{2}\right)$

Because the function extremes $\sin (2 \omega t-\pi / 3)$ are \pm 1 , the maximum values of the force $F_{2}$ (exerted upon the middle conductor), stressed to an attraction (with "+") and, respectively, to a repulsion (with "-") will be:

$$
F_{2 \text { atr } / \text { rep }}= \pm \frac{\sqrt{3}}{2} f_{12}\left(2 I^{2}\right)= \pm 0,866 f_{12}\left(2 I^{2}\right)
$$

\subsubsection{Electrodynamic forces at space conductors settlement}

The settlement of the conductors at the vertices of a equilateral triangle it is indicated [4] for the impedances equalization on the over-head three-phase electric networks or in the case of the short three-phase network (carrying great currents).

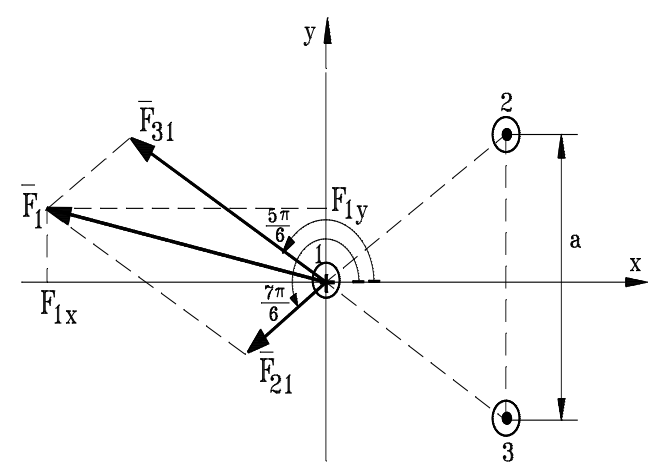

Fig.5. Calculation of force $\mathrm{F}_{1}$ in three-phase circuits with spacial conductors settlement

As example, in Fig.5 have been represented the conductors 1,2 and 3 arranged at the vertices of a equilateral triangle (with the side "a"). This time, the forces are not collinear. Thus, the force $\vec{F}_{21}$ (between the conductors 2 and 1) is acting along the centers direction $\overrightarrow{2} 1$, while the force $\vec{F}_{31}$ (between the conductors 3 and 1) is acting along the centers line $\overrightarrow{3} 1$. Each of these forces has a time variation depending on the alternating currents $\mathrm{i}_{1}, \mathrm{i}_{2}$ and $\mathrm{i}_{3}$, corresponding to the laws:

$$
F_{21}=f_{21} i_{2} i_{1} ; \quad F_{31}=f_{31} i_{3} i_{1}
$$

so that the resulting force $\vec{F}_{1}$ (exerted upon the conductor 1 ) it is obtained by the vector addition (see Fig.5) of the repulsive forces $\vec{F}_{21}$ and $\vec{F}_{31}$ :

$$
\vec{F}_{1}=\vec{F}_{21}+\vec{F}_{31}
$$

For the analytical evaluation of the force $\vec{F}_{1}$ it is considered a reference system xOy (like in Fig.5) and the vectorial equation (16) is projected on the two axes. It will result:

$$
\begin{gathered}
F_{l x}=F_{l} \cos \alpha=F_{2 l} \cos \frac{7 \pi}{6}+F_{3 l} \cos \frac{5 \pi}{6}=-\frac{\sqrt{3}}{2} f_{2 l} i_{2} i_{l}-\frac{\sqrt{3}}{2} f_{3 l} i_{3} i_{l} \\
F_{l y}=F_{I} \sin \alpha=F_{21} \sin \frac{7 \pi}{6}+F_{3 l} \sin \frac{5 \pi}{6}=-\frac{1}{2} f_{2 l} i_{2} i_{1}+\frac{1}{2} f_{3 l} i_{3} i_{l}
\end{gathered}
$$


The resulting force magnitude is established with the formula $F_{1}=\sqrt{F_{1 x}^{2}+F_{1 y}^{2}}$.

Moreover, due to the symmetrical settlement (in space) of the three phase conductors (1,2 and 3), all specific forces will be equal and, consequently, there are accurate the relations:

$f_{12}=f_{21}=f_{23}=f_{32}=f_{31}=f_{13}$

so that similar forces to $\vec{F}_{1}$ (but, with a respective phasedisplacement in time and space) will be acting upon the other two phase-conductors.

Taking into consideration the equalities of relation (18), the perpendicular components $F_{1 x}$ and $F_{1 y}$ become:

$$
F_{1 x}=-\frac{\sqrt{3}}{2} f_{12} i_{1}\left(i_{3}+i_{2}\right) ; \quad F_{1 y}=\frac{1}{2} f_{12} i_{1}\left(i_{3}-i_{2}\right)
$$

With the currents expressions for $i_{1}, i_{2}$ and $i_{3}$ from (6), the components $\mathrm{F}_{1 \mathrm{x}}$ and $\mathrm{F}_{1 \mathrm{y}}$ given by (19) are developed as following:

$$
\begin{gathered}
F_{I x}=\frac{\sqrt{3}}{4} f_{12}(\sqrt{2} I)^{2}[1-\cos 2 \omega t] ; F_{I y}=\frac{\sqrt{3}}{4} f_{12}(\sqrt{2} I)^{2} \sin 2 \omega t \\
\text { If the constant factor } A=\frac{\sqrt{3}}{4} f_{12}(\sqrt{2} I)^{2}
\end{gathered}
$$

emphasized, then the components $F_{1 x}$ and $F_{1 y}$, varying with time, can be written as the compact form:

$F_{l x}=F_{l x}(t)=A-A \cdot \cos 2 \omega t ; F_{l y}=F_{l y}(t)=A \cdot \sin 2 \omega t$

Accordingly, the force magnitude $\mathrm{F}_{1}$ becomes:

$$
F_{1}=\sqrt{{F_{1 x}}^{2}+{F_{1 y}}^{2}}=2 A|\sin \omega t|
$$

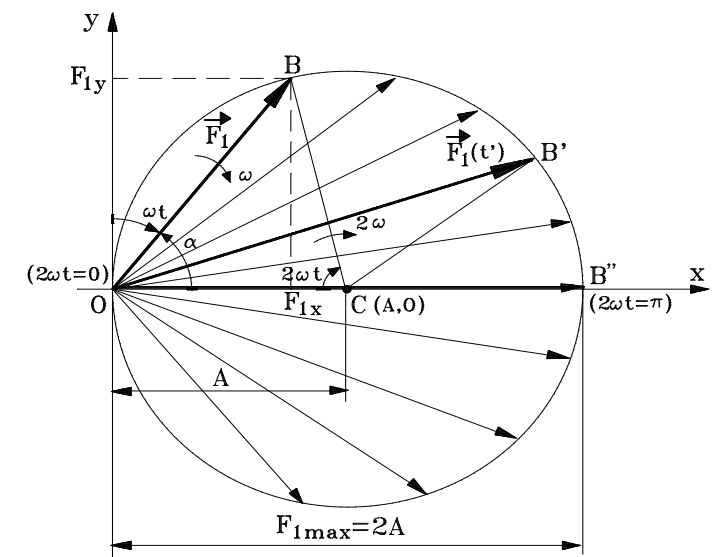

Fig.6. Locus of end-point of force $F_{1}$

From physical considerations, because the components $F_{1 x}$ and $F_{1 y}(21)$ are harmonic varying with time (with the same pulsation $2 \omega$, but on perpendicular directions) it results that the vector peak $\vec{F}_{1}$ (variable with time and space) will describe a circle of radius $\mathrm{R}=\mathrm{A}$, with the center on the axis Ox, in the point $\mathrm{C}(\mathrm{A}, 0)$, like in Fig.6.

From the analysis of Fig.6 one could emphasize that the force $F_{1}$ is varying with time both in magnitude and direction, and its maximum value $\mathrm{F}_{1 \max }=\mathrm{OB}^{\prime \prime}$ is:

$$
F_{1 \text { max }}=0,866 f_{12}(\sqrt{2} I)^{2}
$$

\section{Case study. Power transformer busbars electrodynamic strength under sudden short-circuit conditions. Numerical simulation model and results}

Nowadays, an electric power system encompasses electric equipment that are in operating correlations. For instance, the electric connection circuits of power plants, based on fossil fuels as well as renewable sources, entail generator circuit-breakers (GCBs) at the generator terminals. A generator circuit-breaker is located between the generator and the main step-up transformer, this location influencing the operating conditions since GCBs are significantly more difficult to apply to some operating regimes than classical network circuit-breakers [3-4,9].

The configuration of the key fault current encountered by the generator breaker (GB) is represented in Figure 7, and details are as follows [9]:

- $\quad$ Faults at location $\mathrm{K}_{1}$ are called Generator (G)source faults or Generator-fed faults (which can be insulated or grounded three-phase and twophases short-circuits);

- Faults at location $\mathrm{K}_{2}$ are called System-source faults or Transformer (MT)-fed faults (which can be insulated or grounded three-phase and two-phases short-circuits);

- Faults at location $K_{3}$ are called Generator-fed faults, on the high voltage side of the main stepup transformer (which can be insulated or grounded three-phase and two-phases shortcircuits, as well as single-phase short-circuits).

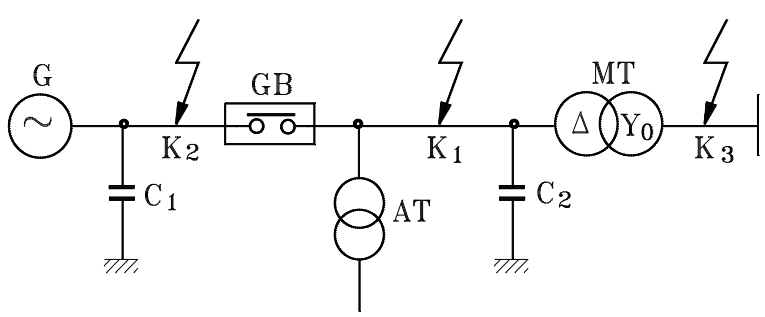

Fig. 7. Possible faults interrupted by a generator breaker GB

To interrupt these kinds of faults, generator circuitbreakers must be capable of interrupting not only the high symmetrical fault current, but also the higher asymmetrical faults currents resulting from high d.c. components of the fault current [3-4,9]. T(2) address meaningfully the operation conditions for the system encompassing the electric generator, the generator circuit-breaker and the main step-up transformer, in this study we analyze the short-circuit located in $\mathrm{K}_{2}$ which is called system-source fault or transformer (MT)-fed fault.

Subsequently we take into consideration that the connection bars in both power transformer primary and secondary might be considered parallel conductors flowed by current. In these connection bars the electrodynamic stresses can be generated, that can be evaluated and visualized graphically for the correct dimensioning of these electrical equipment. These finite 
cross-section bars are elastic mechanical systems insofar as they are not subject to strengths that exceed the elastic limit. Bars can be stacked at two extremities, plain, or fixed to the insulator.

Whether deformation defined by elongation is permanent or non-permanent, it is important that the space between the points defining the electrical potential difference between the two busbars does not exceed the dielectric rigidity of the cooling medium.

High values of electrodynamic strengths are given by short-circuit currents. In rated operating regime the electrodynamic strengths are much lower than those occurring in the short-circuit regime.

This paper is focusing on the short-circuit between two connection bars, meaning a two-phases short-circuit.

In order to built the simulation model using MATLAB and its extensions [10-11], one could consider that the electrodynamic strength under two busbars short-circuit conditions can be expressed in transient regime as the form below:

$S=M_{c} F_{p} F_{d} I_{s c}^{2}\left(e^{-k_{1} t}-\cos k_{2} t\right)^{2}$

where $\mathrm{M}_{\mathrm{c}}$ is a working constant that takes into consideration the magnetic coupling with the magnetic circuit of the power transformer, $F_{p}$ is a positioning constant of busbars, $F_{d}$ is a correction function whose value is derived from the Dwight diagrams, $I_{s c}$ is the estimated short-circuit current, $\mathrm{k}_{1}$ is the working constant of the electric circuit considered with concentrated parameters, and $\mathrm{K}_{2}$ is the shape constant of the signal generated by the numerical simulation scheme.

The positioning constant $F_{p}$ is depending on the ratio $1 / d$, where 1 is the length of busbars, and $d$ is the distance between them.

Subsequently the simulation model is achieved and the simulation results are depicted.

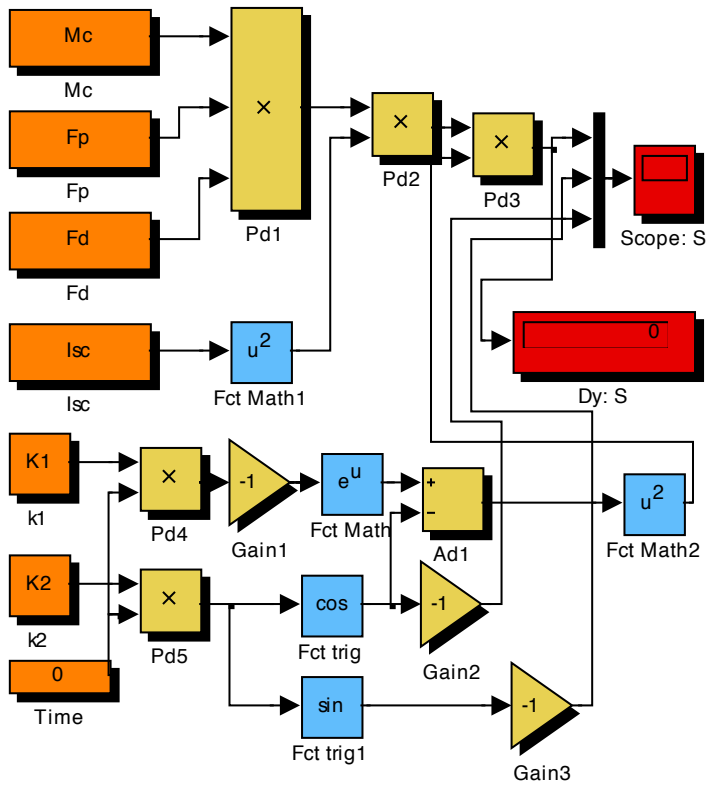

Fig. 8. Numerical simulation model built with MATLAB software Numerical simulation model built with MATLAB software. Input data are set by blocks Mc, Fp. Fd, Isc, K1, K2. Calculation blocks are $\mathrm{Pd}, \mathrm{Ad}, \mathrm{G}$. Calculation functions are defined by blocks Fct. Output blocks Scope S and Dy S generates the working charts,, and displays strengths numerical values, respectively.

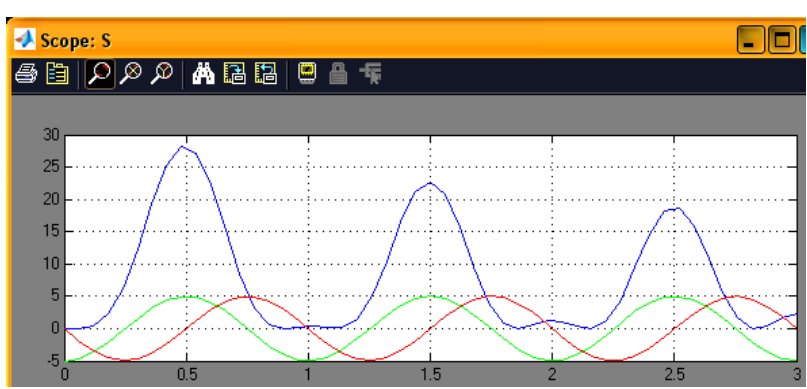

Fig. 9. Simulation of electrodynamic strength in power transformer busbars (blue). Pilot signals: cos (green); $\sin$ (red). $\mathrm{OX}$ axis - time axis $\times 20 \mathrm{~ms}$. OY axis - electrodynamic strength axis. $\mathrm{K} 1=0.25 . \mathrm{K} 2=0.07$.

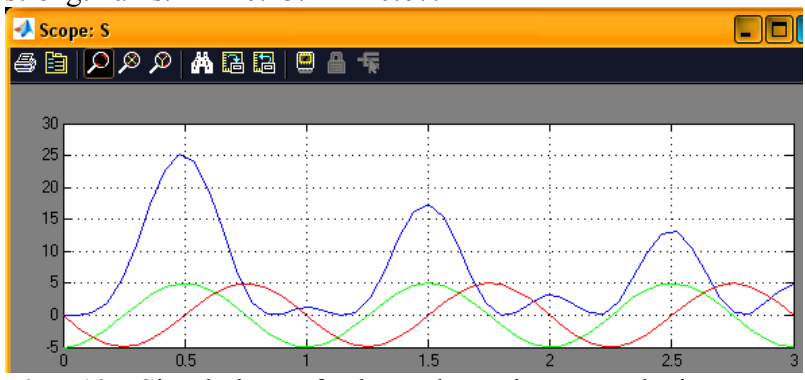

Fig. 10. Simulation of electrodynamic strength in power transformer busbars (blue). Pilot signals: cos (green); sin (red). $\mathrm{OX}$ axis - time axis $\times 20 \mathrm{~ms}$. OY axis - electrodynamic strength axis. $\mathrm{K} 1=0.5 . \mathrm{K} 2=0.08$.

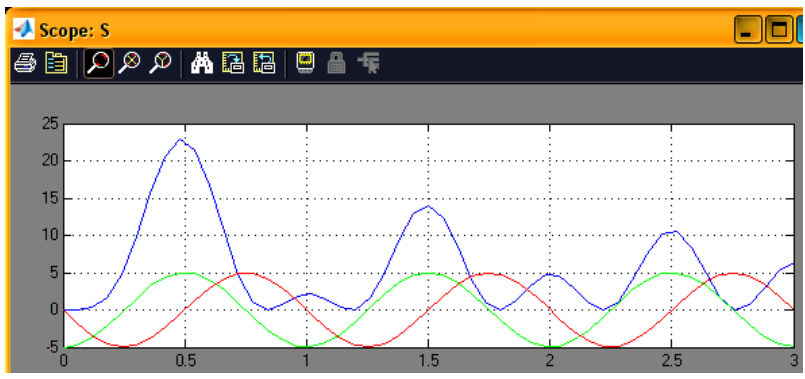

Fig. 11. Simulation of electrodynamic strength in power transformer busbars (blue). Pilot signals: cos (green); sin (red). OX axis - time axis $\times 20 \mathrm{~ms}$. OY axis - electrodynamic strength axis. $\mathrm{K} 1=0.75 . \mathrm{K} 2=0.1$.

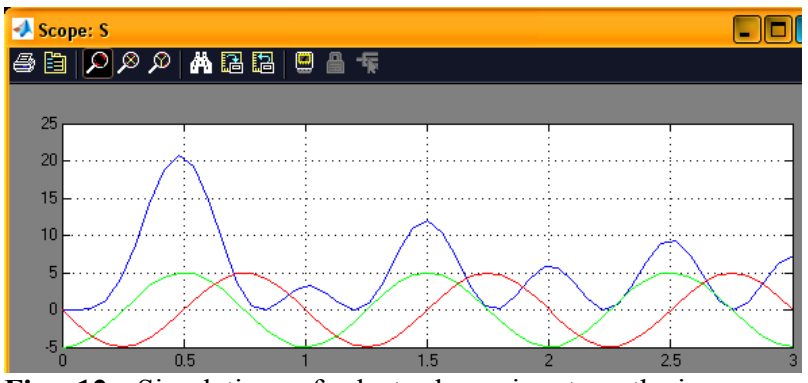

Fig. 12. Simulation of electrodynamic strength in power transformer busbars (blue). Pilot signals: cos (green); sin (red). OX axis - time axis $\times 20 \mathrm{~ms}$. OY axis - electrodynamic strength axis. $\mathrm{K} 1=1 . \mathrm{K} 2=0.2$.

\section{Discussion and Conclusion}

The analytical study highlighted that in the case of the a.c. single-phase circuits, for a r.m.s. value of the short-circuit current $I_{k}$ equal to the current value $I_{d c}$ in a d.c. circuit, the maximum electrodynamic force developed in the a.c. 
circuits can reach values up to 6.5 times greater than the values in the case of the d.c. circuits.

When there are considered the a.c. three-phase circuits with the horizontal phase-conductors settlement, in the same plane, the maximum force exerted upon the middle conductor 2 it is greater than the maximum forces acting upon the lateral conductors 1 , respectively, 3 .

Qualitatively, the analytical results obtained in the analysis of the three-phase circuits forces in the steadystate short-circuit regimes are precise, too, in the case of the transient short-circuit regimes, when the three-phase currents values exceed hundreds times the values of the steady-state short-circuits.

In the case of the three-phase circuit with a space conductors settlement, one can highlight that from numerically, the maximum electrodynamic force has the same value as the maximum of the force exerted upon the middle conductor, when the phase-conductors are horizontally arranged.

Concerning the transient short-circuit regimes with a high asymmetry, it must be taken into consideration the presence of the current aperiodical component, meaning that the maximum (instantaneous) value of the short-circuit current will increase from the value $\sqrt{2} \cdot I$ up to the peak value $i_{\text {peak }}=\sqrt{2} \cdot I \cdot \chi$. Consequently, the electrodynamics forces magnitude will also increase. Therefore, the electric equipments carrying these currents must be capable to stand the greatest forces developed in any possible type of short-circuit, namely single-phase, two-phases or threephase. This way it is ensured the electrical equipment electrodynamic stability.

This analytical study demonstrated the values of the coefficients which appear in the classic electrodynamic strength formulas of the technical literature.

The case study subsequently considered in this paper also allows important discussion and conclusion. Hence, finite cross-section bars are elastic mechanical systems insofar as they are not subject to strengths that exceed the elastic limit. Bars can be stacked at two extremities, plain, or fixed to the insulator. Whether deformation defined by elongation is permanent or nonpermanent, it is important that the space between the points defining the electrical potential difference between the two busbars does not exceed the dielectric rigidity of the cooling medium. High values of electrodynamic strengths are given by short-circuit currents. In rated operating regime the electrodynamic strengths are much lower than those occurring in the short-circuit regime.

The working parameters on the 4 recordings are defined by the electrical constant of the circuit $\mathrm{K}_{1}$ and by the shape constant of the signal generated by the numerical simulation scheme $\mathrm{K}_{2}$. As known $\mathrm{K}_{2} \leq 1$. In our case the electrodynamic strength presents a harmonic variation overlapping on an exponential decrease, so it is amortized over time.

\section{References}

1. M. Jufer , Électromecanique, Traité d'Électricité, Vol.IX, Presses Polytechniques Romandes, CH1015, Lausanne (1992).

2. I. Coulon, M. Jufer, Introduction à l'électrotechnique (Introduction to Electrotechnics), 5th ed., Piesses Politechniques Romandes, Lausanne (1989).

3. C.A. Bulucea,, D.A. Nicola,. Introducere $\hat{\imath} n$ electrotehnica si echipamente electrice (Introduction to Electrotechnics and Electrical Equipment), SITECH Publishing House, Craiova, Romania (2004)

4. D.A. Nicola, C.A. Bulucea, Electrotehnica, masini si echipamente electrice (Electrotechnics, Electrical Machines and Equipment), SITECH Publishing House, Craiova, Romania (2005).

5. I. Rodstein, Electrical Control Equipment, English translation, Mir Publishers (1974).

6. M. Fogiel M., The Electric Circuits. Problem Solver. A complete Solution Guide to Any Textbook, Research \& Education Association (REA) Piscataway, New Jersey, SUA (1998).

7. N. Gheorghiu, A. Selischi, I.N. Chiuță, G. Dedu, G. Comănescu G, Echipamente electrice (Electrical Equipment), Ed. Did. şi Ped., Bucureşti (1981).

8. Gh. Hortopan, Aparate electrice (Electrical Apparatus), Ed. Did. şi Ped., Bucureşti (1980).

9. C.A. Bulucea, M.A. Rosen, D.A. Nicola, N.E. Mastorakis, C.A. Bulucea, Sustainability, 5, 11611176 (2013)

10. M. Ghinea V. Firețeanu, MATLAB. Calcul numeric. Grafică. Aplicații (MATLAB. Numerical calculation. Graphics. Applications), Editura Teora, București (1995).

11. C. Radu, M.C. Ionaşcu, I. Murărița, Statistică Teoretică (Theoretical Statistics), Editura Universitaria Craiova, (2013). 